\title{
Improved Form Navigation on Mobile Devices
}

\author{
A case study with the Egyptian Agricultural Survey
}

\author{
Karim El Batran \\ Computer and Information Sciences Dept. \\ University of Strathclyde \\ Glasgow, Scotland UK \\ karim.el-batran@strath.ac.uk
}

\author{
Mark Dunlop \\ Computer and Information Sciences Dept. \\ University of Strathclyde \\ Glasgow, Scotland UK \\ Mark.Dunlop@cis.strath.ac.uk
}

\begin{abstract}
- in this paper a study was done regarding improving form control and navigation on mobile devices. An observational study of census counters for the Egyptian Agricultural Survey was conducted. This country-wide survey is currently conducted by professional counters using large (100x35 cm double sided) complex paper forms that require manual transcription. Computerization would be beneficial in terms of accuracy and duplication of effort. However there are considerable challenges in reducing the size of the forms to fit mobile devices. Furthermore counters typically have low technological experience. Based on our observations we developed two prototypes: one using traditional form tabs, the other pan-and-zooming. Results from initial user tests showed the pan-and-zoom interface was both faster and had a lower perceived effort.
\end{abstract}

Keywords- Mobile Devices; HCI (Human Computer Interaction); Usability; LTBU (Low Technological Background User); small-screen devices navigation and Form display.

\section{INTRODUCTION}

A form is a document (printed or electronic) with spaces in which to write or enter data. Forms are used to increase the uniformity and efficiency of handling routine tasks, to ensure that tasks are completed and that information can be found when needed [4]. The quality of digital form interfaces depends primarily upon three factors. One of these factors is the clarity of the design and visual representation of the screens [3]. In the bulk of current form design practice the element, layout, and functionality of user interfaces is intended for casual users who access the forms on an irregular basis and prefer simple interfaces rather than more powerful alternatives. This has led to the adoption of basic point and click interaction model in which navigation and control was accomplished by selecting user interface elements with a pointing device such as a mouse [1]. Today, new techniques are widely used to interact with touchscreen interfaces, particularly on mobile devices, yet the interaction style for forms has not been re-evaluated.

Completing complex forms is both time consuming and error prone with users making incorrect selections and spending considerable time navigating the form. Traditional scrollbars were used in navigating through large forms so that the large information space could fit on screen. Igarashi and Hinckley [5] have identified that one of the major two limitations with using traditional interaction is that users have to shift their focus between the document and the scrollbar. They suggest that this may increase the operational time and may cause a significant attentional overhead. Furthermore, in large documents small scrollbar movements can cause a large movement of the document that disorients users.

To counter this visual blurring and to reduce physical navigational workload, there were two techniques. First, the tabbing technique, which converts the form into several separate parts. Second, pan and zoom interfaces, typical of image viewing applications, view the whole document as one navigable screen $[2,6]$. Successive zooming in and out by the user gradually expands and contracts the form contents while panning changes the section of the form to be displayed. Zooming and panning technique used for document and map navigation tasks have proved to achieve user's acceptance and improvement in the overall performance and are now widespread on tablets and phones. However, their use in complex form filling has yet to be fully explored.

Here we initially introduce a case study of the Egyptian Agricultural Census. We then describe an observational study conducted with census counters and two versions of a tablet based solution, one using traditional tabbing and one pan-andzoom. Finally, we discuss user studies conducted to assess two iPad interfaces for navigating and completing the complex forms. Finally, we conclude and discuss future work.

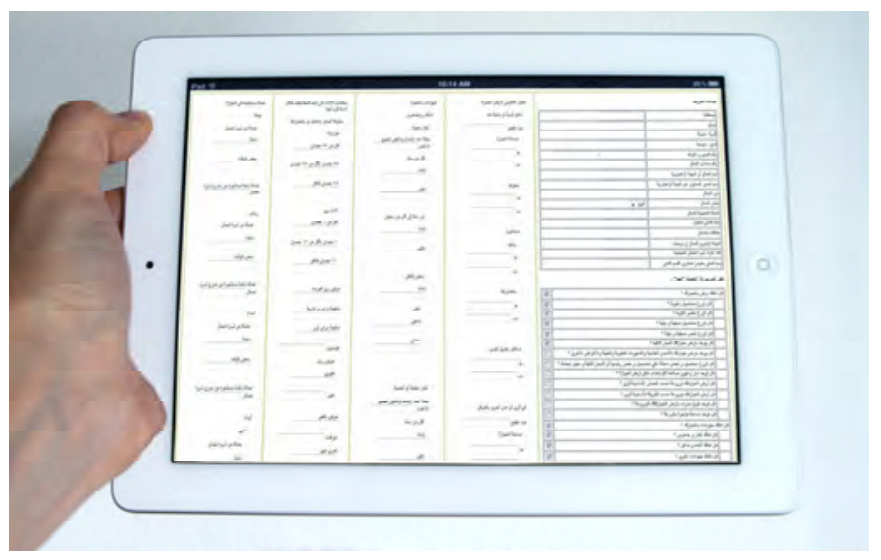

Fig. 1. View of the entire form using zoom technique. 


\section{CASE Study}

The Egyptian Agricultural Census is a comprehensive inventory of the national economic structure of agriculture in a specific time period (one agricultural year). It is done under the supervision of the state by collecting accurate information about agricultural production units (agricultural holdings such as number of agricultural holdings, areas, uses, geographically characterization and categorization, status of irrigation and drainage, number of livestock and poultry, agricultural machinery and farmers), the features and characteristics of such possessions. It is also used to measure the direction and rates of change compared to the results of previous censuses and current statistics, in order to discern characteristics and development of the economic structure in the agricultural sector. The most important purpose of implementing agricultural census is to rely on its results as a basis for planning by economists, social researchers and also draw a final picture that describes the relationship between different economic sectors. In next section, we introduce an observational study conducted on the Egyptian Agricultural Census.

\section{The OBSERVATIONAL STUDY}

The EAC (Egyptian Agricultural Census) deals with an inventory of all possessions located in villages and cities of the Nile Valley, as well as possessions located in desert governorates and land belonging to the reconstruction organization.
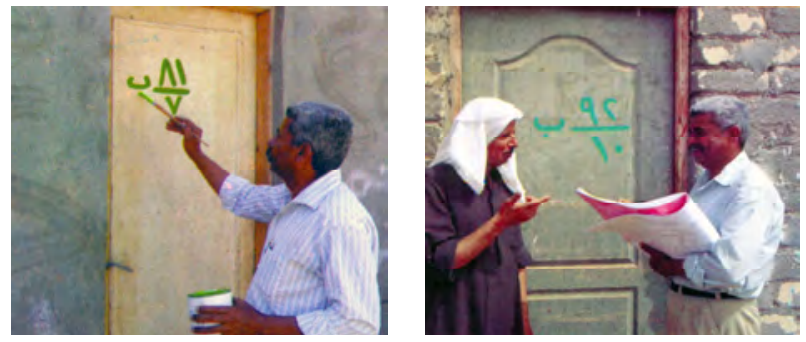

Fig. 2. Numbering buildings and census interviews while completing paper forms.

As shown in figure 2, the census is conducted through personal interviews done by counters with the holders in their place of residence after numbering the buildings to identify them easily in villages and small towns. However, because in big cities numbering is difficult, the counters follow the method of inventory by walking through agricultural basins and taking landholders addresses in order to reach them.

The EAC is conducted through two phases. First phase, a sketch is set at the beginning for all villages and towns nationwide, the buildings are then numbered and a visit is made to all the holders to collect initial data. Second phase, a visit is made to all the families to collect more precise data including personal information and other detailed data regarding their possession. All gathered data are written in paper forms. The preliminary stage of the EAC contains four steps; issuing executive decisions to conduct the EAC, preparing the census budget and adopting it, choosing the field supervisors, engineers, agents and participants in the governorates and training workers on the agricultural census. A trial census is done in order to measure the efficiency of the forms used, revise the method of data collection, identify problems and obstacles and observe the response of holders to questions on the census. This trial census was held in three selected governorates representing the regions of Upper and Lower Egypt.

There are certain obligatory rules that must be adhered to by counters and the head-of-counters. First, the allocation of one head-of-counters to each five counters. Second, taking into account the equitable distribution among counters and headsof-counters in areas of operation. Third, the revision of the numbering that took place in the first phase, buildings not mentioned should be documented by writing a notice. Forth, buildings must be visited in the same order of the records and all the holders contained in the records must be visited. Finally, the heads-of-counters accept records after being reviewed precisely.

The following flow chart illustrates how the process of the census and data collection is done.

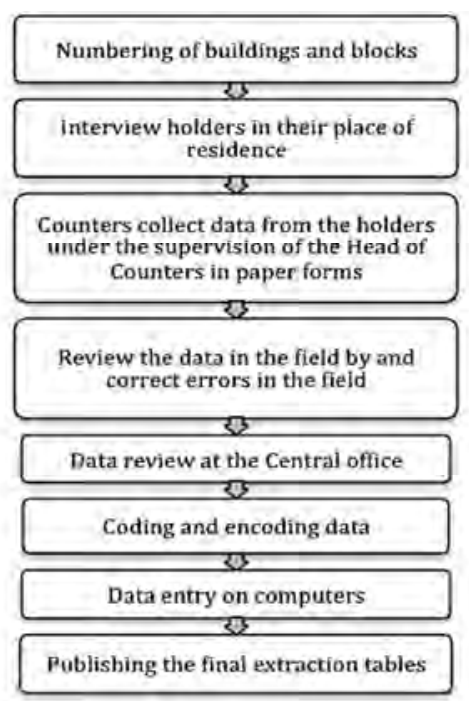

Fig. 3. Egyptian Agricultural Census Process Flow.

In our observations and discussions with senior counters, we found most counters could be categorized as Low Technological Background users (LTB). LTB users are categorized as having low to zero levels of experience dealing with computerized devices such as PCs, laptops, tablets, smartphones and/or cell phones. For reference, the percentage of computer users was approximately 23\% in Egypt in July 2010. This percentage increased to $42.78 \%$ in July 2013 [7].

\section{COMPUTERIZATION CHALLENGE}

Although, there is technological progress in informationgathering systems and data collection, the Egyptian Ministry of Agriculture and Land Reclamation still collects the data manually on large (double sided 100x35 cm) paper forms contained in books of 40 forms, as in the right image in figure 1. The Ministry then transfers the books physically to the 
headquarters, and then enters the data to a database using "Data Entry" employees separate from the counters - primarily because of the low technical background typical of the counters. This double entry has been identified as affecting the accuracy of data and increasing the cost of the census. However, introducing more computerized data collection using electronic forms leads to interesting questions such as: What is the attitude of the LTB users towards computerizing the forms? How will the change of size from large forms to tablets affect interaction? In particular will the need to navigate through the form affect external working memory of the counters? Do pan and zoom techniques better support their working memory than traditional tabbing interfaces? Will computerizing the process help in decreasing the overall time, increasing its accuracy and decreasing the cost?

\section{INITIAL COMPARATIVE USER STUDIES}

Our observational studies highlighted the complexity of forms completed, their physical size and the low technical background of census counters. Here, we compare using two interfaces, one based on tabbing and one on zooming, for completing forms on tablets to assess which interface style is easier to use for the counters given their experience.

\section{A. System Description}

The system is aimed to minimize the time, which the counter takes interviewing the holder. With our system, counters initially answer a number of yes/no questions to generate the form elements required. Afterwards, the counter begins filling and submitting the form.

\section{B. System Design}

The form is designed using HTML5 and JavaScript to be displayed on an Apple iPad 2 using the standard Safari browser (9.7" screen with resolution of $1024 \times 768)$. It is based on two techniques: selecting and typing. The form is composed of a combination of checkboxes and textboxes and was designed to closely resemble the original paper version in structure and individual components

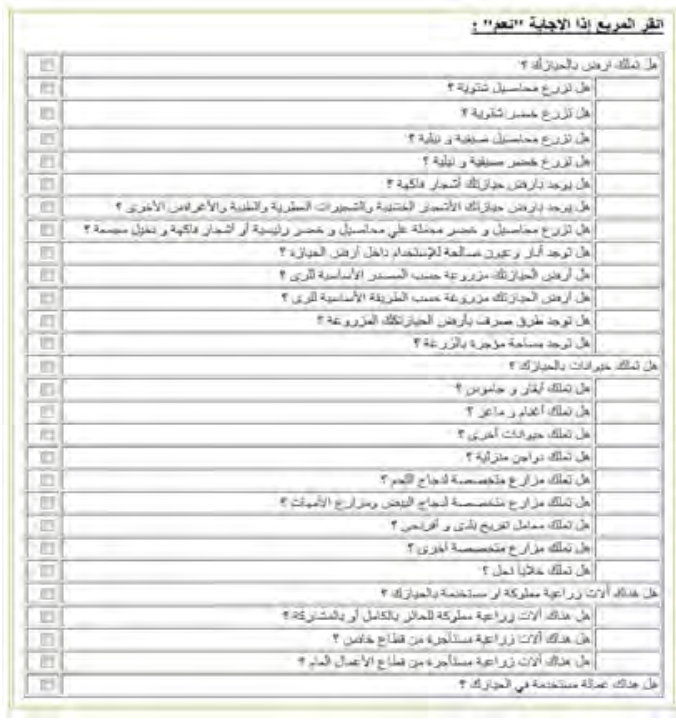

Fig. 4. 28 (yes/no) questions.
The paper Agricultural Census form is divided into 5 main sections containing 666 fields to be filled in with data (not all fields are mandatory). Section 1 contains 16 mandatory questions regarding the holder's information; Section 2, data regarding the possession of land (420 fields); Section 3 data about livestock and animals (100 fields); Section 4 concerns Agricultural machinery (63 fields); and the final section, information regarding labor used (57 fields). These five sections are reflected in the electronic form. The first section is visible to enter in the data. The other four are optional and initially hidden, set to visible according to the selection of their checkbox on the overview section.

First, the counter enters the essential data of the holder's personal information. The counter asks the holder $28 \mathrm{yes} / \mathrm{no}$ questions as shown in figure 4; these questions generate the entire form. There are four main questions representing the remaining four main sections of the form. Each main question contains a number of sub questions, which represents a set of required information to be given by the holder and filled by the user (counter). This part represents the first section of the form. Subsequently, the counter begins filling in the form. Later, after editing, updating fields and making sure that all fields are completed and correct, the user submits the form.

\section{User Evaluation}

We conducted a usability study in which we compared the zoom and tab interfaces in terms of task-completion times and workload. We recruited 20 counters. Reflecting the typical counter our subjects were all male, aged 25-42 and bachelors graduates. Their fields of study varied greatly, but not computer science; none were familiar with tablets or extensive PC users, but all were familiar with the original paper version of the Agricultural Census.

As mentioned before, we are comparing two navigation techniques; the pan and zoom technique against the traditional tabbing technique. In the pan and zoom technique, the method of zooming in on the screen image includes sliding your thumb and index finger outward across the screen.

This method allows the user to zoom in on specific areas in the form for edit or update. Restoring the entire form requires from the user to slide his thumb and index finger inward across the screen in a pinching motion. This enables the user to view the whole form in one screen. In the traditional tabbing technique, the form is viewed and divided into multi-tabs view. Each section is displayed in a separate tab. This method allows the user to view each section separately.

Figures 1 and 5 show the two interface versions. Users were asked to complete two scenario forms on each device. The dependent variables were task-completion time (in seconds), interface preference, and subjective workload. The workload was measured with the NASA Task Load Index questionnaire (NASATLX). Tasks and order of presentation of interfaces were randomly balanced. 


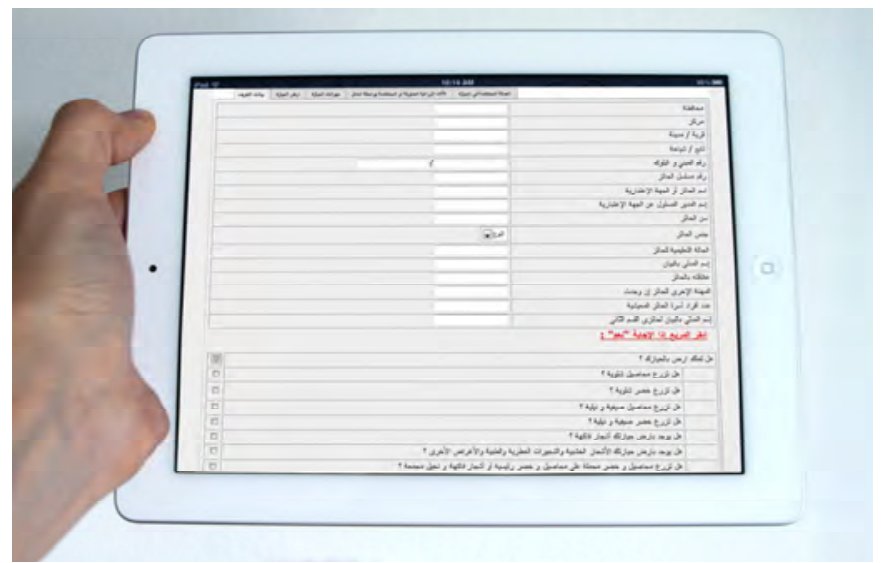

Fig. 5. View of the form using tabbing technique.

\section{Results}

Our results show that it took our participants about 31 minutes on average to complete the tasks using zooming technique, but 35 minutes on average using tabbing technique. This showed significantly lower task completion time $(\mathrm{p}<0.05$, paired t-test, $\mathrm{df}=19$ ) as shown in figure 6 . Moreover, NASA TLX forms were completed after each interface and showed indicatively lower workload for effort $(\mathrm{p}<0.1)$ using the zooming technique.

Finally, users were asked at the end of the study "Did you prefer the computerized version of the form, which navigation technique do you prefer and why". All participants were satisfied using the electronic form. Fourteen of the twenty users favored the zooming technique. Their comments were clear regarding the ability of viewing the whole form rather than hopping through tabs. A couple of them suggested adding the ability of the user to determine the uncompleted sections or fields in the form.

While the results show that the zooming technique was significantly faster than tabbing and we attempted to have representative users, the results are based on a relatively small sample of participants doing artificial tasks in a laboratory setting. Longer field-based trials are needed to confirm the results and to assess fully whether a zooming based tablet application could improve on paper-based forms.

\section{CONCLUSION}

We have presented a study of professional form use through an observational study of census counters. We then developed two tablet-based forms to replace paper forms. We introduced a new approach to improve form navigation for form filling on mobile devices using pan-and-zoom techniques.

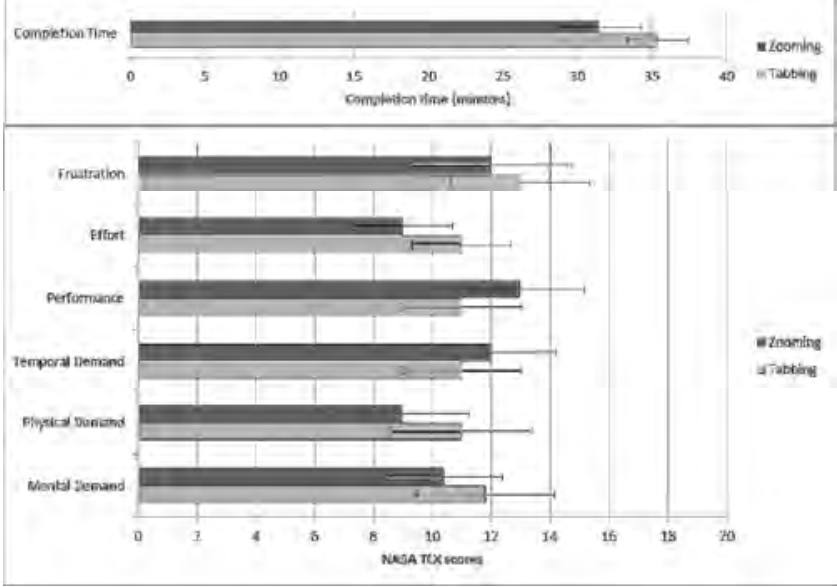

Fig. 6. Time and NASA TLX results.

Using zooming gave users the ability to view the whole form rather than hopping through tabs. In our controlled user tests with users of lower technological background than traditional tablet users, we showed significantly reduced input time and perceived effort with pan-and zoom interaction.

Further user studies are now planned to accurately model form filling process using zooming techniques, in particular to assess the use of zoomed out views in assessing formcompletion status and use of zoomed out views as external working memory.

\section{ACKNOWLEDGMENT}

We would like to thank the participants in both our observational studies and tablet trials.

\section{REFERENCES}

[1] Ballard, A., Shafer, P. \& Sherwood, T. "Customization of tab-order functionality in internet applications", US Patent, no. 7111243, (2006).

[2] Bederson, B., Hollan, J., Perlin, K., Meyer, J., Bacon, D. \& Furnas, G. "Pad++: a zoomable graphical sketchpad for exploring alternate interface physics", Proc. ACM UIST, (1994).

[3] Buxton, W.A.S., \& Baecker, R.M. Human-computer interaction: a multidisciplinary approach, Morgan Kaufmann, (1987).

[4] Dayao, A.B., Rafieyan, K., Lee, P.J., Graveman, R.F., Yacobi, Y., \& Addeo, E.J., "SuperForms: A Security-Enhanced Smart Electronic Form Management System", Proc. IEEE Global Telecommunications, vol. 2, (1990).

[5] Igarashi, T. \& Hinckley, K. "Speed-dependent automatic zooming for browsing large documents", Proc. ACM UIST 2000, (2000).

[6] Perlin, K. \& Fox, D. "Pad: an alternative approach to the computer interface", Proc. ACM SIGGRAPH, (1993).

[7] Ministry of communication and Information Technology (MCIT). "Egypt ICT Sector Indicators in brief August 2013", (2013). 